\title{
Fecal Occult Blood Test (FOBT) Overuse
}

\author{
Melissa R. Partin, $P h D^{7}$, Adam A. Powell, $P h D^{7}$, and James F. Burgess Jr PhD ${ }^{2}$ \\ ${ }^{1}$ Center for Chronic Disease Outcomes Research, Minneapolis VA Health Care System, Minneapolis, MN, USA; ${ }^{2}$ Center for Organization, \\ Leadership and Management Research, VA Boston Healthcare System, Boston, MA, USA.
}

J Gen Intern Med 28(5):611

DOI: $10.1007 / \mathrm{s} 11606-013-2397-1$

(c) Society of General Internal Medicine 2013

$\mathrm{T}$ he authors' reply — We appreciate Dr. Federman's comments and agree that computerized clinical reminder (CCR) and performance measurement systems may play an important causal role in overuse of Fecal Occult Blood Testing (FOBT) in the Veterans Health Administration (VHA).

At most VHA facilities, colorectal cancer screening CCRs are programmed to appear 2-3 months before the tests are due. As Dr. Federman notes, by encouraging the early completion of reminders, the screening interval for some patients may be inappropriately compressed. However, this would not explain why, among patients in our data set with a prior colonoscopy, the average window between colonoscopy and FOBT was 3.1 years-almost 7 years shorter than the recommended interval. We suspect many prior colonoscopies are being missed entirely by CCR algorithms. We used 30 different CPT and ICD-9 codes to identify colonoscopies. It is unclear how many VHA colorectal cancer screening CCRs (which are programmed at the facility level) utilize such a comprehensive list. Efforts to improve the VHA colorectal cancer screening CCRs to minimize FOBT overuse should verify whether all prior colonoscopies are being captured in the system.

Dr. Federman also describes how the VHA performance measurement system may have the unintended consequence of promoting overuse by incentivizing high screening rates without creating disincentives for overuse. An important next step in the evolution of VHA's performance measurement system is the development of overuse measures to address this issue. This will be challenging because, in addition to identifying whether or not a procedure was done, overuse measures must determine the presence or absence of contraindications for a procedure. In our study, we examined screening tests that were done too soon following a prior test, but other contraindications for screening FOBTs include (1) gastrointestinal symptoms or bleeding that warrant diagnostic colonoscopy, and (2) presence of late stage disease or other comorbidities that make it unlikely that the patient will live long enough to benefit from screening. Given the considerable variability across VHA facilities in how the requisite information is entered into the system, designing and implementing an overuse measurement system that can feasibly and reliably identify these contraindications on a national scale is a formidable task. However, this should not deter VHA or other healthcare systems from pursuing the challenge. Even if initially flawed, such measures are likely to stimulate further investigation into the causes of overuse, and improvements in coding protocols that will yield more accurate measures in the future.

Corresponding Author: Melissa R. Partin, PhD; Center for Chronic Disease Outcomes Research, Minneapolis VA Health Care System, One Veterans Drive, Minneapolis, MN 55417, USA (e-mail: Melissa.partin@va.gov).

Published online March 9, 2013 